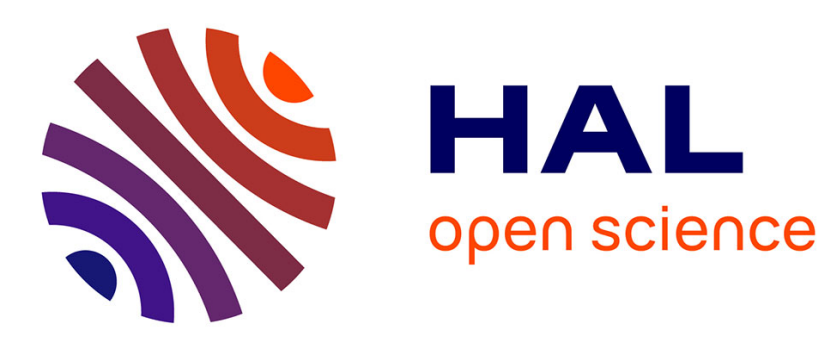

\title{
Tidal bore effect in heavy ion collisions
}

Henri Orland, R. Schaeffer

\section{To cite this version:}

Henri Orland, R. Schaeffer. Tidal bore effect in heavy ion collisions. Journal de Physique Lettres, 1976, 37 (12), pp.327-331. 10.1051/jphyslet:019760037012032700 . jpa-00231304

\section{HAL Id: jpa-00231304 https://hal.science/jpa-00231304}

Submitted on 1 Jan 1976

HAL is a multi-disciplinary open access archive for the deposit and dissemination of scientific research documents, whether they are published or not. The documents may come from teaching and research institutions in France or abroad, or from public or private research centers.
L'archive ouverte pluridisciplinaire HAL, est destinée au dépôt et à la diffusion de documents scientifiques de niveau recherche, publiés ou non, émanant des établissements d'enseignement et de recherche français ou étrangers, des laboratoires publics ou privés. 


\title{
TIDAL BORE EFFECT IN HEAVY ION COLLISIONS
}

\author{
H. ORLAND and R. SCHAEFFER
}

\author{
Service de Physique Théorique \\ Centre d'Etudes Nucléaires de Saclay \\ $\mathrm{BP}^{\circ}{ }^{\circ}$ 2, 91190 Gif-sur-Yvette, France
}

(Reçu le 23 août 1976, révisé le 15 octobre 1976, accepté le 16 octobre 1976)

\begin{abstract}
Résumé. - Nous prédisons une nouvelle classe de phénomènes durant la collision de deux ions lourds, dans un domaine d'énergie allant de 40 à $200 \mathrm{MeV}$ par nucléon. La dépendance en densité de l'interaction effective dans la matière nucléaire engendre un potentiel à un corps répulsif dans la région de recouvrement des deux noyaux. Le bord de cette partie répulsive, qui se déplace à la vitesse de la particule incidente, repousse violemment les nucléons du noyau cible. Ce phénomène de raz de marée produit de la matière nucléaire qui se propage en avant du projectile, peut créer des pions ou être éjectée.
\end{abstract}

Abstract. - Between the very high energy regime $(E>200 \mathrm{MeV} / \mathrm{N})$ where the energy deposited by the projectile into the target nucleus propagates behind the incoming particle at the velocity of sound and the low energy regime $(E<40 \mathrm{MeV} / \mathrm{N})$ where partial equilibrium might be reached, we predict the appearance of a new class of phenomena. The density dependent single particle well has a repulsive edge in the double density region. This moving edge pushes the target nucleons in the foward direction at very high velocity (twice the velocity of the incoming particles). This tidal bore produces nuclear matter which propagates ahead of the projectile, may create pions or be ejected.

1. Introduction. - At extremely high energies $(1 \mathrm{GeV} / \mathrm{N})$ one expects the particles in the fragment to see each other individually and therefore twobody collisions to dominate. There is, however, a limiting energy below which the nucleons react cooperatively, i.e. the dynamics can be described in terms of Hartree-Fock fields or single particle wells. Nevertheless the question to determine these limits and to describe the role one or two-body collisions respectively play is still open. The promising calculations $[1,2]$ using the time-dependent single particle model in order to describe low energy reactions between heavy ions lead us to consider it for the study of the phenomena at energies larger than $40 \mathrm{MeV} / \mathrm{N}$. This model, where one calculates the corrections to the sudden approximation [1] should be quite a good starting point in this case. The single particle well $U$ a nucleon experiences when two ions come to close contact is [1]

$$
\begin{aligned}
U=U_{\mathrm{A}} & +U_{\mathrm{B}}+ \\
& +\frac{2}{\gamma}\left(1-\sqrt{1-\gamma U_{\mathrm{A}}}\right)\left(1-\sqrt{1-\gamma U_{\mathrm{B}}}\right)
\end{aligned}
$$

where $U_{\mathrm{A}}$ and $U_{\mathrm{B}}$ are the single particle wells of the two undisturbed fragments. The last (repulsive) term arizes from the density dependence of the nuclear force and $\gamma$ can be directly obtained from the parameters of the Skyrme force. In order to display schematically the various collision regimes, we take simply one dimensional, $50 \mathrm{MeV}$ deep square wells (Fig. 1a). In the double density region, with the usual Skyrme parameters, $U$ changes from attractive to repulsive (Fig. 1b). The repulsive barrier which thus appears in the region of overlap reflects the nucleons in the fragments. Again for simplicity let us assume that the potential vanishes in the high density region. A nucleon in the target $\left({ }^{1}\right)$ with velocity $v\left(v_{\mathrm{F}}>v>-v_{\mathrm{F}}\right)$ is reflected by the edge of the well moving at velocity $V$ if in the frame attached to the well its kinetic energy $\frac{1}{2} m(v-V)^{2}$ is smaller than $E_{\mathrm{B}}$ and its velocity $v-V$ negative since the nucleon has to propagate towards the edge. Let us define $v_{B}$ such as

$$
\frac{1}{2} m v_{\mathrm{B}}^{2}=E_{\mathrm{B}}
$$

So, if $V>V_{\mathrm{cr}}=v_{\mathrm{B}}+v_{\mathrm{F}}$ there are no reflected particles at all. This is the critical velocity above

$\left({ }^{1}\right)$ For symmetry reasons we can consider only the target nucleons in order to describe this phenomenon, but in the numerical estimates all particles will be taken into account. 


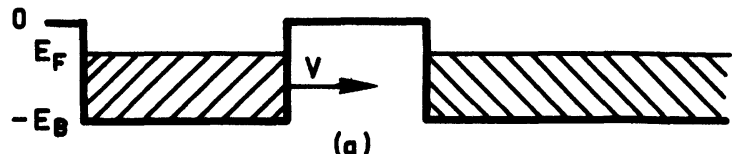

(a)

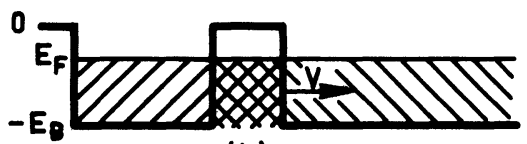

(b)

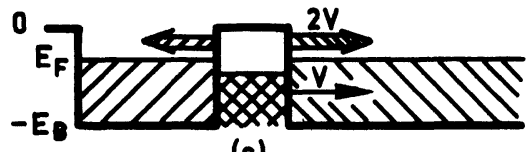

(c)

FIG. 1. - Schematic display of a well with velocity $V$ colliding with a slab of nuclear matter at rest. The hatched areas show the nucleons in the wells. The depth of the well is $E_{\mathrm{B}}(>0)$ and $E_{\mathrm{F}}=K_{\mathrm{F}}^{2} / 2 m$ is the Fermi energy measured from the bottom of the well. In $(a)$ we show he wells before the collision, in $(b)$ the collision at $V>V_{\text {cr }}$ (see text) and in (c) at $V<V_{\text {cr. }}$. In the two last cases, the barrier in the overlapping region is due to the density dependence of the nuclear forces. The hatched arrows in $(c)$ show the nucleons reflected by the moving edges of the wells. The reflected nucleons of the well, which was initially at rest, move roughly with a velocity $2 V$.

which only two-body collisions play a role. (Nevertheless, some of the effects due to these collisions may still be included in a suitably defined Hartree-Fock field). The corresponding energy of the projectile is $E_{\mathrm{cr}} \approx 180 \mathrm{MeV} / \mathrm{N}$. For $V_{\mathrm{cr}}>V>v_{\mathrm{B}}$, the reflected particles are those with velocity $v_{\mathrm{F}}>v>v_{\mathrm{F}}-w$, with

$$
V=V_{\mathrm{cr}}-w .
$$

For a one-dimensional Fermi sea, the fraction reflected is $w / 2 v_{\mathrm{F}}$. The velocity $v-V$ of the particle in the frame attached to the moving edge is changed into $V-v$ after reflection. So it becomes in the laboratory frame :

$$
v^{\prime}=2 V-v
$$

which may be much larger than the velocity of the incoming projectile. Let us study these two regimes in more detail.

2. Collisions above the critical energy. - Above the critical energy, we consider the energy loss of the incoming particle as provided by two-body collisions. This is the domain where shock waves $[3,4,5]$ in the usual sense arise : energy is deposited into the target nucleus and the perturbation propagates at the velocity of sound, behind the projectile if nuclear matter is transparent enough. For simplicity, we shall not consider any other phenomena in this energy region which is essentially examined here for comparison with the lower energy domain. The energy loss $\varepsilon$ of a projectile nucleon due to its collision with a target nucleon at rest is the average recoil energy

$$
\varepsilon=\left\langle\frac{q^{2}}{2 m}\right\rangle
$$

where $q$ is the momentum transfer, and assuming isotropic cross-sections is equal to half the lab energy of the incoming nucleon, i.e.

$$
\varepsilon \sim \frac{1}{2} E \text {. }
$$

The projectile is thus slowed down by the constant frictional force

$$
F_{\mathrm{N}}=\varepsilon \rho \sigma_{\mathrm{N}}, \text { i.e. } \mathrm{d} V / \mathrm{d} t=F_{\mathrm{N}} / m
$$

where $\rho$ is the density of nuclear matter, $m$ the nucleon mass and $\sigma_{\mathrm{N}}$ the total elastic nucleon-nucleon crosssection. Note that this force is independent of the fragment's masses. We have omitted a factor $1-E_{\mathrm{F}}^{2} / E^{2}$ in $F_{\mathrm{N}}$ (obtained approximately by taking the average in (5) for $q>k_{\mathrm{F}}$ only, due to the reduction of the free nucleon-nucleon cross-section because of the Pauli principle which diminishes the available phase-space). At the energies we consider, this factor is nearly unity. Above the pion threshold

$$
\left(E>E_{\pi} \sim 280 \mathrm{MeV} / \mathrm{N}\right),
$$

$F_{\mathrm{N}}$ should be replaced by $F_{\mathrm{T}}=F_{\mathrm{N}}+F_{\pi}$ with :

$$
F_{\pi} \sim m_{\pi} \rho \sigma_{\pi}
$$

when $\sigma_{\pi}$ is the total pion production cross-section.

However, $F_{\pi}$ is much smaller than $F_{\mathrm{N}}$ in this energy range $\left(\sigma_{\pi} \sim 4 \mathrm{mbarn}\right)$. So, the main dependence of $F_{\mathrm{T}}$ is the factor $\varepsilon \sim V^{2}$ (the energy loss is proportional to the available energy) which, however saturates at high energy when the nucleon-nucleon cross-sections become forward peaked. So, for the energies above the nucleon-nucleon pion threshold $E_{\pi}$ we have simply chosen $\varepsilon \approx 250 \mathrm{MeV}$. This is somewhat too large for $E_{\pi}>E>E_{\mathrm{cr}}$. In the latter energy range (as well as for $E_{\mathrm{cr}}>E>E_{\mathrm{F}}$ in the next section, where anyway other dissipative phenomena occur) we shall take $\varepsilon \sim 100 \mathrm{MeV}$. This leads to $F_{<}=110 \mathrm{MeV} / \mathrm{fm}$ for $E>E_{\pi}$ and $F_{>} \sim 40 \mathrm{MeV} / \mathrm{fm}$ for $E<E_{\pi}$. The actual values are of course a smooth interpolation between the two, the discontinuity at $E$ due to pion emission being only of about $10 \mathrm{MeV} / \mathrm{fm}$. The total distance the projectile travels is given by

$$
R=\int_{E_{\mathrm{F}}}^{E_{\mathrm{LAB}}} \frac{\mathrm{d} E}{F(E)} .
$$

This is plotted as the solid line in figure 2. The total number of pions produced while a projectile is slowed down to $E_{\pi}$ from its initial energy $E$ is

$$
n_{\pi}=\frac{1}{2} \rho_{\mathrm{L}} \rho \sigma_{\pi}\left(E-E_{\pi}\right)^{2} / F_{>}^{2}
$$




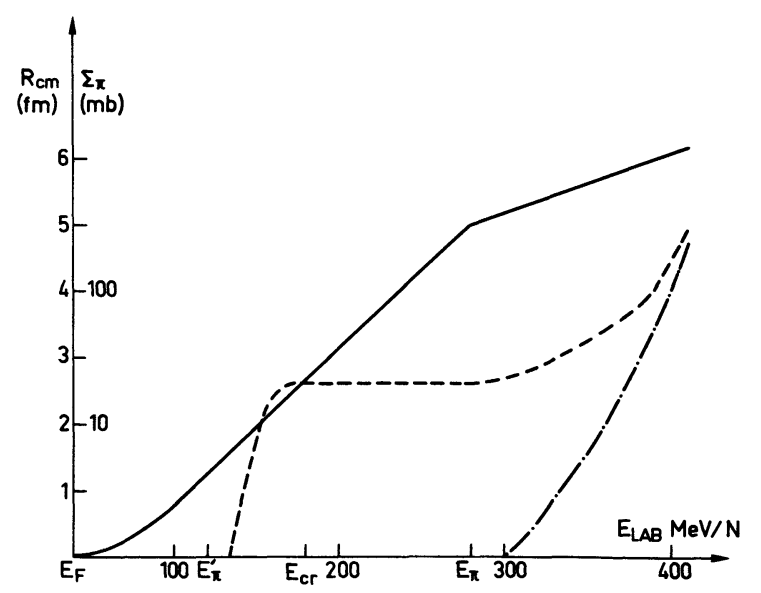

FIG. 2. - Full line : Distance $R$ a heavy ion propagates in the target as a function of its incoming energy,$E_{\mathrm{LAB}}$. The kink at $E_{\mathrm{LAB}}=E_{\pi}$ is not only due to the pion production, as explained in the text.

Dashed line : Total pion production cross-section $\Sigma_{\pi}\left(E_{\mathrm{LAB}}\right)$ for an incoming ion with energy $E_{\mathrm{LAB}}$. The normal pion production cross-section for $E_{\mathrm{LAB}}>E_{\pi}$ is $\Sigma_{\pi}\left(E_{\mathrm{LAB}}\right)-\Sigma_{\pi}\left(E_{\pi}\right)$. The remaining part of the cross-section, for $E_{\mathrm{cr}}>E_{\mathrm{LAB}}>E_{\pi}$ is due to abnormal pion production due to shock phenomena around $E_{\mathrm{LAB}} \sim E_{\mathrm{cr}}$. All these cross-sections should be scaled by a factor

$$
\left(\frac{A}{12}\right)^{2 / 3} \frac{\pi b^{2}}{100 F m^{2}}
$$

when $A$ is the mass of the smaller fragment and $b$ the maximum impact parameter for which the fragments hit each other.

Dash-dotted line : Total pion production cross-section without the tidal bore effect.

Fermi motion has been neglected for all the pion cross-section calculation. It would smooth out all the curves.

where $\rho_{\mathrm{L}}$ is the linear density in the one-dimensional well $\left(\rho_{\mathrm{L}} \approx \frac{2}{3} \pi R_{\mathrm{A}}^{2} \rho\right.$, where $R_{\mathrm{A}}$ is the radius of the smaller nucleus, taken as $1.2(12)^{1 / 3}$ for the numerical estimates). The corresponding cross-section, assuming all projectiles with an impact parameter smaller than $b$ hit the target is

$$
\Sigma_{\pi}(E)=\pi b^{2} n_{\pi}
$$

Eq. (10) and (11) are plotted as the dot-dash curve in figure 2. We have neglected the Fermi motion of the nucleons in order to obtain (10). This will be discussed later on.

3. Collisions below the critical energy. - Below the critical energy, the moving edge which meets $\rho_{\mathrm{L}} V \mathrm{~d} t$ target nucleons during the time $\mathrm{d} t$, reflects a fraction

$$
\mathrm{d} n_{\mathrm{R}}=w / 2 v_{\mathrm{F}} \rho_{\mathrm{L}} V \mathrm{~d} t
$$

of nuclear matter which is projected ahead of the projectile with an average velocity.

$$
\bar{V}^{\prime}=2 V-v_{\mathrm{F}}+\frac{1}{2} w,
$$

and a spread $w$, corresponding to a maximum (for $\left.V \approx V_{\text {cr }}\right)$ energy of $423 \mathrm{MeV} / \mathrm{N}(434 \mathrm{MeV} / \mathrm{N}$ with relativistic kinematics). It is slowed down by the two-body collisions. Hence, the average velocity at time $t$ of the wave reflected at $\tau$ is, for $t>\tau$

$$
\bar{V}^{\prime \prime}(\tau, t)=\bar{V}^{\prime}(\tau)-F / m(t-\tau)
$$

In general, this wave propagates faster than the projectile (Fig. 3), i.e. $V^{\prime \prime}>V$. There is a succession of such waves emitted at different times $\tau$, which propagate behind each other since $\bar{V}^{\prime \prime}(\tau, t)$ is a decreasing function of $t$ (Fig. 3b). Defining $t_{\mathrm{cr}}$ as the time at which $V$ reaches the critical velocity $V_{\text {cr }}$, for $t>t_{\mathrm{cr}}$ there is therefore a big tidal bore of reflected matter propagating at a large velocity in front of the projectile (Fig. 3). It produces pions as long as $V^{\prime \prime}>V_{\pi}$. This happens for projectile energies ranging from $E_{\text {cr }}$ down to $E_{\pi}^{\prime}=120 \mathrm{MeV} / \mathrm{N}$, i.e. well below the threshold of normal pion production. This is therefore a mechanism for coherent pion production at very low incident energies. It might therefore be observed, and distinguished from the direct mechanism.

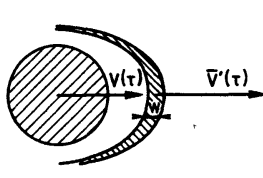

()

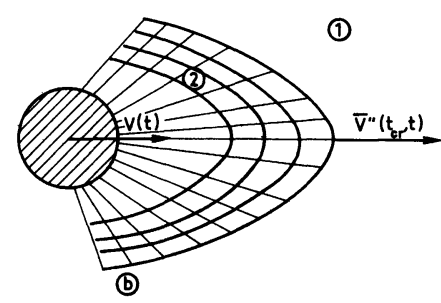

FIG. 3. - Three-dimensional picture of a small projectile entering nuclear matter at $V<V_{\mathrm{cr}}$ a) Wave of reflected matter at time $\tau$, with velocity $\bar{V}^{\prime}(\tau)>V(\tau)$ and spread $\left.w . b\right)$ Schematic picture at $t>t_{\mathrm{cr}}$ of the succession of waves reflected at all the times $t>\tau>t_{\mathrm{cr}}$ which build up the tidal bore effect. The label (1) denotes the unperturbed region and (2) the region over which the reflected matter flows.

The fraction of nuclear matter that has kinetic energy larger than $E_{\mathrm{B}}$ when it reaches the other edge of the target well will be projected out, and the remaining part provides some additional heating of the target nuclear matter. The estimate of the average number of ejected nucleons depends, however, crucially on the three dimensional geometry of the system and cannot be done in the framework of the present study (Note however that a large fraction of the pion produced by any of the processes we have considered should come out since the pion absorption crosssection is still small).

Before being able to give some semi-quantitative estimates, we have to consider the projectile motion in more detail. The momentum it communicates to the reflected target nucleons during the time $\mathrm{d} t$ decreases its velocity by the amount

$\mathrm{d} w=\frac{w \rho_{\mathrm{L}}}{2 v_{\mathrm{F}} \mu_{\mathrm{r}}}\left(V_{\mathrm{cr}}-w\right)\left(2 V_{\mathrm{cr}}-v_{\mathrm{F}}-\frac{3}{2} w\right) \mathrm{d} t$

where $\mu_{\mathrm{r}}$ is the reduced mass of the system. 
Since we shall restrict ourselves to $V_{\text {cr }}>V>v_{\mathrm{F}}$, i.e. $v_{\mathrm{B}}>w>0$, we can use an average value of $w$, i.e. $w \sim v_{\mathrm{B}} / 2 \approx v_{\mathrm{F}} / 2$ in order to linearize (14) to

$$
\mathrm{d} w / \mathrm{d} t=w / \tau+F_{<} / m, \quad \text { with } 1 / \tau \sim 2 \rho_{\mathrm{L}} v_{\mathrm{F}} / \mu_{\mathrm{r}}
$$

where the velocity loss $F_{<} / m$ due to two-body collisions has been added. As a function of time, $w$ is thus given by

$$
w=F_{<} / m \tau\left(\mathrm{e}^{t / \tau}-1\right) .
$$

For small times $(t<\tau)$, the velocity loss is still due to the two-body collision term, i.e. $w \sim F_{<} / m t$. For $t>\tau$, the projectile is stopped very quickly due to the increasing amount of nuclear matter it has to push forward. The time $\tau$ is of the order of $10 \mathrm{fm} / \mathrm{c}$, and from figure 2 it can be seen that an incoming particle with energy $E=E_{\text {cr }}$ is stopped within two Fermi. The matter it has projected ahead has an energy somewhat below $420 \mathrm{MeV} / \mathrm{N}$. The distance the nucleons propagate with a sufficient energy to produce pions, $R_{\pi}$, is given by eq. (9), integrated between the limits $E=\frac{1}{2} m\left(2 V_{\mathrm{cr}}-V_{\mathrm{F}}-\frac{1}{2} w(t)\right)^{2}$ and $E_{\pi}$. In this energy range $\varepsilon$ should be taken to be about $175 \mathrm{MeV}$, leading to $F \sim 75 \mathrm{MeV} / \mathrm{fm}$. So it propagates over a distance of about $2 \mathrm{fm}$ before it has been slowed down below the pion threshold. The number of pions produced is proportional to the number of nucleons reflected, the distance they propagate times the rate of production per unit length

$$
\mathrm{d} n_{\pi}^{\prime}=2 \sigma_{\pi} \rho_{\mathrm{L}} R(t) \mathrm{d} n_{\mathrm{R}} .
$$

When integrated, this leads to a quite sizeable total pion production cross-section.

$$
\Sigma_{\pi}^{\prime}=\pi b^{2} n_{\pi}^{\prime}
$$

of the order of $20 \mathrm{mb}$ for $\pi b^{2}=100 \mathrm{fm}^{2}$. The dashed curve in figure 2 is the sum of $\Sigma_{\pi}$ and $\Sigma_{\pi}^{\prime}$.

4. Discussion. - Let us discuss in some detail the main assumptions we have made. All the phenomena described below $E_{\mathrm{cr}}$ depend on the assumption that the usual density dependence of the nuclear forces as given by the Skyrme force is still valid at twice the nuclear matter density. This is still beyond our present stage of knowledge of heavy ion reactions. But, whatever the hight $E_{\mathrm{B}}$ of the repulsive barrier is, the critical energy is given by $E_{\mathrm{cr}}=\left(\sqrt{E_{\mathrm{B}}}+\sqrt{E_{\mathrm{F}}}\right)^{2}$. The existence of shock waves (or not), and the observation of the ejected matter or produced pions as a function of the incident energy will actually provide us with this information. Also, we have assumed that the particles reflected at the edges of the well obey classical dynamics. Although qualitatively true, the actual velocity of the particles that experience an external well is smaller than the classical prediction [1], for velocities smaller than $v_{\mathrm{F}}$. Here all reflected particles have velocities larger than $v_{\mathrm{F}}$. If this slowing down should persist for the reflected particles we have considered, there may simply be no pions emitted at all for $E<E_{\mathrm{cr}}$. Some matter could still be ejected, but in the worst case the reflected wave of nuclear matter would only heat up the target. More involved Hartree-Fock or hydrodynamical calculations (including temperature) could answer this question.

The numbers we have given depend somewhat on the estimate of the energy loss $\varepsilon$ due to two-body collisions. Should this loss be larger (it might be) than our estimate, say be $\varepsilon^{\prime}$, the normal pion production would decrease as $\left(\varepsilon / \varepsilon^{\prime}\right)^{2}$ whereas the tide race production decreases only by the amount $\varepsilon / \varepsilon^{\prime}$. If the energy loss is much larger $\left(\varepsilon / \varepsilon^{\prime} \sim \frac{1}{4}\right)$ the incomingion as well as the reflected matter is stopped very rapidly and compression as described by Sobel et al. [5] will occur. This would then be a one fluid description of the collision whereas we advocate as in ref. [6] rather for a multi-fluid description due to the larger mean free path we predict.

We have also neglected the Fermi-motion of the nucleons in the estimate of pion production, for $E>E_{\pi}$ as well as for $E<E_{\mathrm{cr}}$. This important correction can be included as outlined in reference [7]. This will extend the normal pion production threshold (with a small cross-section) almost down to $E \sim E_{\mathrm{F}}$, and the abnormal threshold up to $E>E_{\pi}$. Nevertheless, the main strength should still be concentrated at the energies we predict. The best would be to observe the pion production cross-section as a function of decreasing incident energy. The normal pion production cross-section will decrease exponentially below $E=E_{\pi}$ and the tidal bore production should manifest itself as a $20 \mathrm{mb}$ kink on this exponential tail. It may also be interesting to look for pion production below $100 \mathrm{MeV} / \mathrm{N}$ where the tidal bore mechanism should contribute much more than the normal mechanism.

Quantum-mechanical effects also will play some role. Barrier penetration effects will weaken the reflected flux at $E<E_{\mathrm{cr}}$. But also above-barrier reflection will produce some reflected matter at $E>E_{\text {cr. }}$. This also should result on a somewhat smoothed out effect. But even in the case the incoming well produces an attractive barrier, due to its sharp edge, quantum mechanical reflection will create exactly the same tidal bore although at a weaker intensity. In a more usual language this corresponds to many particle-hole excitations by the edge of the incoming well into the continuum and pion production by the excited particles.

5. Conclusion. - Using a very simple and naive model, we have displayed schematically some the features of heavy ion collisions at energies ranging from 40 up to $200 \mathrm{MeV} / \mathrm{N}$. The most interesting is the possible coherent production of pions at very low incident energies. But even the ejection of nuclear matter and the heating of the nuclear matter ahead 
of the projectile even at velocities larger than the velocity of sound is worth a careful investigation. It might be important for a hydrodynamical description of heavy ion collisions, and lead to some infor- mation on the density dependence of the nuclear forces under unusual conditions, far from equilibrium. We would like to thank P. Bonche for discussions, and G. F. Bertsch for useful comments and criticisms.

\section{References}

[1] BertsCh, G. F. and SChaefFer, R., to be published in Nucl. Phys.

[2] Bertsch, G. F., Blaizot, J. P., SchaefFer, R. and Vichniac, G., in preparation.

[3] Chapline, G., Johnson, M., Teller, E. and Weiss, M., Phys. Rev. D 8 (1973) 4302.
[4] Scheid, W., Muller, H. and Greiner, W., Phys. Rev. Lett. 32 (1974) 741

[5] Sobel, M., Siemens, P., Bondorf, J. and Bethe, H., Nucl. Phys. A 251 (1975) 502

[6] BeRTSCH, G. F., Phys. Rev. Lett. 34 (1975) 697.

[7] BERTSCH, G. F., to be published. 